\title{
The Dose Distribution from Iridium-192 Source on Cervical Cancer Brachytherapy by Manchester System Using Monte Carlo Simulation
}

\author{
F. Kurniati ${ }^{1}$, F. P. Krisna ${ }^{1}$, Junios ${ }^{2 *}$, F. Haryanto ${ }^{1}$ \\ ${ }^{1}$ Faculty of Mathematics and Natural Sciences, Institut Teknologi Bandung, \\ Jl. Ganesa 10, Bandung, West Java 40132, Indonesia \\ ${ }^{2}$ Institut Kesehatan Prima Nusantara Bukittinggi, \\ Jl. Kusuma Bhakti No.99 Gulai Bancah Bukittinggi, West Sumatera 26122, Indonesia
}

\section{ARTICLE INFO}

Article history:

Received 11 December 2020

Received in revised form 17 June 2021

Accepted 27 August 2021

Keywords:

Brachytherapy

CT image

Ir-192 source

Manchester system

Monte carlo simulation

\begin{abstract}
A B S T R A C T
One treatment for cervical cancer is to use radioactive sources that directly target the cancer cell called brachytherapy. This study is aimed to determine dose distribution at phantom pelvis using the DOSXYZnrc Monte Carlo code. The phantom was derived from a CT scan image of the DICOM-type pelvis with a size of $50 \times 50 \times 28.8 \mathrm{~cm}$ obtained from Santosa Kopo Hospital. The source used was Ir-192, which makes an asymmetrical beam with a size of $0.45 \times 0.09 \times 0.09 \mathrm{~cm}$. Monte Carlo simulation was performed to determine the dose distribution of the Ir-192 source on cervical cancer CT images based on the Manchester system. The Monte Carlo simulation was divided into two models with distance variations on the applicator. Model A used TPS data with a distance between sources of $0.9 \mathrm{~cm}$, while model B had a distance between sources of $0.5 \mathrm{~cm}$. The distribution of dose resulting from the Monte Carlo simulation was analyzed and compared with TPS data. The results showed that at the range of $50 \%$, dose distribution in model A reaches the end of $3.9 \mathrm{~cm}$. When compared to the range of $50 \%$ dose distribution at the TPS results that reaches the point of $4 \mathrm{~cm}$, it produces a deviation value of $2.5 \%$, which is still within the tolerance range. Model A and Model B provide different dose distribution. In model $\mathrm{B}$, it reaches $3.86 \mathrm{~cm}$, resulting in a deviation of $1.02 \%$, which is still within the tolerance range. The resulting $\gamma$-index value for the $50 \%$ dose distribution was 2.26 , while the whole area's GPR value was $94.13 \%$. This indicates a difference in dose distribution between the two models. Therefore, the smaller the distance between the sources, the shorter the dose distribution range with relatively more uniform dose distribution.
\end{abstract}

(C) 2021 Atom Indonesia. All rights reserved

\section{INTRODUCTION}

The number of new cancer cases in Indonesia based on data from the International Agency for Research on Cancer (IARC) estimates that 207.210 patients die of as many as 348.809 cases. Cervical cancer has many death cases, as many as 32.469 people or $17.2 \%$ of the total cases [1]. Cervical cancer is caused by the infection of the Human Papilloma Virus (HPV). One of the available treatments of cervical cancer is by using ionizing radiation and radioactive sources, known

\footnotetext{
${ }^{*}$ Corresponding author.

E-mail address: juniosmsi@gmail.com

DOI: https://doi.org/10.17146/aij.2021.1102
}

as radiotherapy. These two methods of radiotherapy are teletherapy and brachytherapy $[2,3]$.

In brachytherapy, radioactive seeds are placed in or near the tumor to provide a high radiation dose to the tumor while reducing radiation exposure to the surrounding healthy tissue $[4,5]$. The radioactive source used in this study is Iridium-192, which is classified as a high dose rate (HDR) brachytherapy treatment [6].

Iridium-192 is inserted into the cancer cells through the applicator using intracavitary brachytherapy technique. The system used for the placement of radioactive sources is the Manchester system (Patterson-Parker system). The 
Manchester system method is to implant a radioactive source into the body, designing uniform dose distribution [7]. The treatment planning system's accuracy influences brachytherapy treatment in determining the dose distribution in patients. The treatment planning system (TPS) will produce dose distribution to maximize tumor control and minimize usual tissue complications. According to AAPM REPORT, a less than $5 \%$ dose for a tumor will reduce the tumor probability control by $15 \%$ or more, while an excess dose of $5 \%$ for a tumor will cause an increase in the dose in healthy tissue so that it exceeds the tolerated dose [6]. Whereas, based on the International Commission for Radiation Units and Measurements, the main cause of failure of radiation therapy treatment is related to the loss of geographic targets due to inaccurate target delineation and dosimetric variations of more than $3 \%$ [7]. For early-stage tumors, it has been reported that a $1 \%$ increase in overall beam delivery accuracy results in a $2 \%$ increase in cure rate [8]. Therefore, it is crucial to accurately predict the dose when calculating the radiation dose. In this study, a simulation was carried out to find out the dose distribution of the Ir-192 source in cervical cancer brachytherapy using the Monte Carlo method (EGSnrc). This method models the microscopic process by following each simulated particle [9]. Monte Carlo is the most accurate method of calculating the dose distribution since every physical particle of the material will be calculated using the sampling method of a probabilistic distribution function.

\section{METHODOLOGY}

The phantom pelvis used in this study was originated from a CT scan image of the DICOMtype pelvis with a resolution $512 \times 512$ pixels, obtained from Santosa Kopo Hospital. Each pixel's value from the CT image informs the object's attenuation coefficient expressed in the CT number or Hounsfield Unit (HU). The network density in the CT image expressed in HU was then interpolated into a 3D voxel matrix and converted to the phantom's material type and thickness through the ct-create program. The ct-create converted a CT image into a virtual phantom by adjusting the phantom's voxel size. The phantom size of the CT image used in this study is $50.0 \times$ $50.0 \times 28.8 \mathrm{~cm}$. The voxel size used in the phantom is shown in Table 1 .
Table 1. Number and size of voxel on the phantom.

\begin{tabular}{cccc}
\hline Axis & $\begin{array}{c}\text { Number } \\
\text { of Voxel }\end{array}$ & $\begin{array}{c}\text { Voxel } \\
\text { Size }\end{array}$ & voxel limit value \\
\hline $\mathrm{X}$ & 125 & $0,4 \mathrm{~cm}$ & $-25,000 \mathrm{~cm}$ to $+25,000 \mathrm{~cm}$ \\
$\mathrm{Y}$ & 125 & $0,4 \mathrm{~cm}$ & $-40,300 \mathrm{~cm}$ to $+9,700 \mathrm{~cm}$ \\
$\mathrm{Z}$ & 72 & $0,4 \mathrm{~cm}$ & $-16,150 \mathrm{~cm}$ to $+12,849 \mathrm{~cm}$ \\
\hline
\end{tabular}

Based on Table 1, there is a shift in the number and size of voxels in the virtual phantom, where the number of voxels read by the ct-create program is $512 \times 512 \times 145$ voxels with a voxel size of $0.09 \times 0.09 \times 0.19 \mathrm{~cm}$. This shift is due to the virtual phantom voxel size setting adjusts to the maximum number of voxels and the voxels' minimum size in the ct-create mortran code files. Furthermore, converting the Hounsfield Unit into a phantom constituent materials type and density through interpolation forms a standard CT ramp on the ct-create. Standard CT ramp on ct-create distinguishes four kinds of phantom constituent materials: air, lungs, soft tissue, and bone. These materials are differentiated based on the HU range contained in the CT image. The ct-create program output, a file with the egsphant extension, will be automatically stored in the CT file folder and is ready for use in DOSXYZnrc.

The radioactive source used in this study was Iridium-192 (Ir-192). The Ir-192 source geometry in the Monte Carlo simulation was modeled as an asymmetric block measuring at $0.45 \times 0.09 \times$ $0.09 \mathrm{~cm}$, with an energy range of $0.065-0.885 \mathrm{MeV}$. In this study, the Ir-192 sources were placed one by one at the specified coordinates ignoring the material at Ir-192, only showing the same shape in terms of the source size. The source model used is ISOURCE $=6$ Uniform Isotropically Radiating Parallele piped in DOSXYZnrc Volume.

The applicator's size was adjusted to the applicator size at TPS, namely the length of the intrauterine tube (tandem) of $60 \mathrm{~mm}$ and the regular colpostat tube (ovoid) with a diameter of $20 \mathrm{~mm}$. The simulation of the Ir-192 source consists of two stages. The first stage of the simulation was carried out based on the Manchester system adjusted to TPS data called model A. The second stage of the simulation optimized the distribution of the Ir-192 dose with a distance of $0.5 \mathrm{~cm}$ between sources, called model B. The Matlab software was employed to combine the .3ddose output data, which was then analyzed through the isodose curve and compared with the TPS results' isodose curve. 


\section{Model A}

In model A, the Ir-192 source was inserted into the applicator with the source position following the original position in the TPS data. The position of Ir-192 in the applicator is shown in Fig. 1.

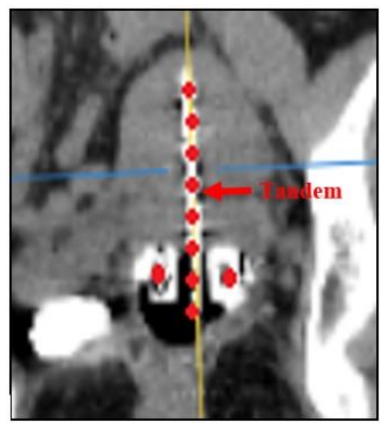

(a)

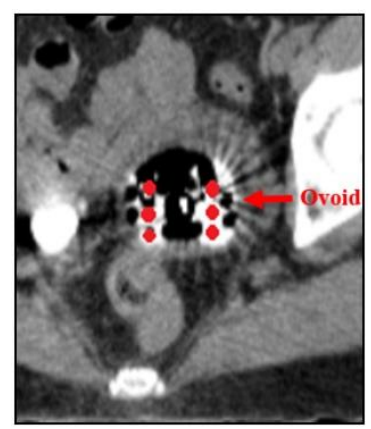

(b)
Fig. 1. Position of Ir-192 source in the applicator for model A; (a). axial view; (b). coronal view.

In Fig. 1, there are 14 Ir-192 sources consisting of $8 \mathrm{Ir}-192$ sources in the tandem (Fig. 1.a) and 3 Ir-192 sources in each ovoid (Fig. 1.b). For more details, the position of the Ir-192 is provided in Table 2.

Table 2. Position of Ir-192 source on the applicator for model A.

\begin{tabular}{ccccccc}
\hline \multirow{2}{*}{$\begin{array}{c}\text { Source } \\
\text { Location }\end{array}$} & \multicolumn{3}{c}{ Minimum Position } & \multicolumn{3}{c}{ Maximum Position } \\
\cline { 2 - 7 } & Xinl & Yinl & Zinl & Xinu & Yinu & Zinu \\
\hline \multirow{6}{*}{ Ovoids } & 0.17 & -14.9 & -0.42 & 0.26 & -14.9 & 0.03 \\
& 0.11 & -15.5 & -0.60 & 0.20 & -15.4 & -0.15 \\
& 0.05 & -15.9 & -0.83 & 0.14 & -15.8 & -0.38 \\
& 2.29 & -15.1 & -0.63 & 2.44 & -15.1 & -0.18 \\
& 2.27 & -15.6 & -0.82 & 2.38 & -15.5 & -0.37 \\
& 2.23 & -16.0 & -1.05 & 2.32 & -15.9 & -0.60 \\
\hline \multirow{6}{*}{ Tandem } & 1.51 & -18.3 & 4.07 & 1.60 & -18.2 & 4.52 \\
& 1.47 & -17.9 & 3.16 & 1.56 & -17.8 & 3.61 \\
& 1.43 & -17.5 & 2.25 & 1.52 & -17.4 & 2.69 \\
& 1.39 & -17.1 & 1.34 & 1.49 & -16.9 & 1.78 \\
& 1.36 & -16.7 & 0.43 & 1.45 & -16.6 & 0.87 \\
& 1.32 & -16.3 & -0.48 & 1.41 & -16.2 & -0.03 \\
& 1.28 & -15.8 & -1.39 & 1.37 & -15.7 & -0.94 \\
& 1.25 & -15.4 & -2.30 & 1.34 & -15.3 & -1.85 \\
\hline
\end{tabular}

Based on Table 2, one Ir-192 source on the Z-axis has the initial position expressed in Zinl, and the final part in Zinu. The distance between sources is defined as the distance between the initial part of the first source (Zinl-1) and the initial position of the subsequent source (Zinl-n). The distance between the Ir-192 sources in tandem for model A on the $\mathrm{Z}$-axis is $0.9 \mathrm{~cm}$.

\section{Comparison of the Ir-192 source position for model A and model B}

The distance between the Ir-192 sources based on TPS data on the Z-axis is $0.9 \mathrm{~cm}$, while the maximum distance between radioactive sources based on the Manchester system is $0.5 \mathrm{~cm}[10,11]$. According to AAPM REPORT, the the error of dose distribution rate at the distance of $0.5 \mathrm{~cm}$ between sources was calculated to be less than $2 \%$. Therefore, in the second simulation stage, optimization of the dose distribution of the Ir-192 source with a distance between the sources of $0.5 \mathrm{~cm}$ is called model B. The difference in the $\operatorname{Ir}-192$ source position in tandem for model A and model B is shown in Fig. 2.

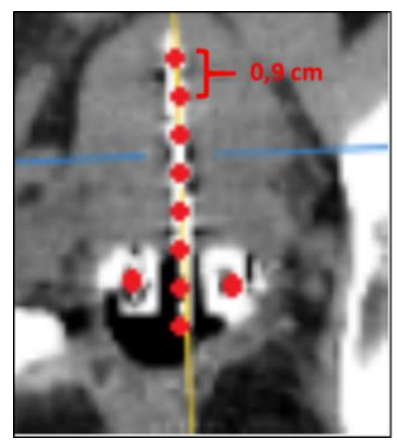

(a)

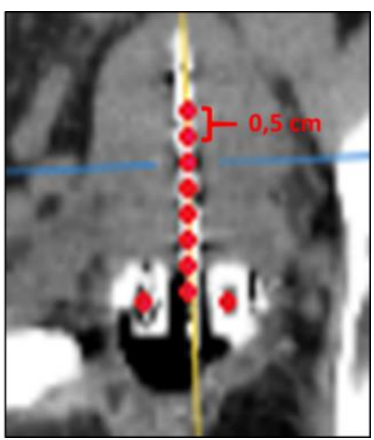

(b)
Fig. 2. Position of Ir-192 source in tandem; (a). Model A; (b). Model B.

Based on Fig. 2, the Ir-192 source placement in model A and model B has a different position. In the tandem at the direction of the $\mathrm{Z}$-axis, namely model A, the distance between Ir-192 sources was set at $0.9 \mathrm{~cm}$, while model $\mathrm{B}$ has the distance between Ir-192 sources of $0.5 \mathrm{~cm}$. The seeds in the ovoids did not change the position due to the distance between the sources is $<0.5 \mathrm{~cm}$, so their location were the same as the original position in model A. For more details, the position of the Ir-192 source in tandem for model B is showing in Table 3.

Table 3. Position of Ir-192 source in tandem for model B.

\begin{tabular}{ccccccc}
\hline \multirow{2}{*}{$\begin{array}{c}\text { Source } \\
\text { Location }\end{array}$} & \multicolumn{3}{c}{ Minimum Position } & \multicolumn{3}{c}{ Maximum Position } \\
\cline { 2 - 7 } & Xinl & Yinl & Zinl & Xinu & Yinu & Zinu \\
\hline \multirow{6}{*}{ Tandem } & 1.51 & -18.3 & 4.07 & 1.60 & -18.2 & 4.52 \\
& 1.47 & -17.9 & 3.16 & 1.56 & -17.8 & 3.61 \\
& 1.43 & -17.5 & 2.25 & 1.52 & -17.4 & 2.69 \\
& 1.39 & -17.1 & 1.34 & 1.49 & -16.9 & 1.79 \\
& 1.34 & -16.7 & 0.43 & 1.45 & -16.6 & 0.88 \\
& 1.32 & -16.2 & -0.49 & 1.41 & -16.2 & -0.03 \\
& 1.28 & -15.8 & -1.40 & 1.37 & -15.7 & -0.94 \\
& 1.25 & -15.4 & -2.30 & 1.35 & -15.3 & -1.85 \\
\hline
\end{tabular}


Based on Tables 2 and 3 , the different position of the Ir-192 sources in tandem not only occur at the $\mathrm{Z}$-axis direction but also at the $\mathrm{X}$ and $\mathrm{Y}$-axis directions. In model $\mathrm{A}$, the $\mathrm{X}$-axis sources' distance is $0.038 \mathrm{~cm}$, while on the $\mathrm{Y}$-axis direction is $0.4 \mathrm{~cm}$. In model $\mathrm{B}$, the distance between sources on the $\mathrm{X}$-axis is $0.021 \mathrm{~cm}$, while on the $\mathrm{Y}$-axis is $0.2 \mathrm{~cm}$. The spread between sources on the $\mathrm{X}$ and $\mathrm{Y}$ axes for model A and model B is still within the tolerance limits of the distance between sources based on the Manchester system. The output data from this simulation is in the form of a .3ddose file, which then will be analyzed using the Matlab program to determine the dose distribution of the Ir-192 source through isodose curves and gamma index.

In all simulations, it was considered that $1.9 \times$ $10^{8}$ incident particles and $1.25 \times 10^{7}$ particles are reaching the scoring plane. Furthermore, the electron cutoff energy (ECUT) was $0.7 \mathrm{MeV}$, and photon cutoff energy (PCUT) was $0.01 \mathrm{MeV}$. The Random Number Generator (RNG) is user-defined to obtain random values [12-15].

Eq. (1) was used to determine the difference between the TPS result and simulation result.

$$
\Delta=\frac{\text { TPS result-simulation result }}{T P S \text { result }} \times 100 \%
$$

The analysis of the gamma index will be carried out using Matlab. The gamma pass rate (GPR) represents the presentation of the number of tracks with a gamma index value less than 1 relative to the number of all pixels or voxels. Eq. (2) was used to determine the GPR.

$$
(\mathrm{GPR})=\frac{\text { range of } \gamma \leq 1}{\text { range of } \gamma} \times 100 \%
$$

The GPR value greater than $95 \%$ indicates that the two compared methods have the same dose distribution [16].

\section{RESULTS AND DISCUSSION}

Pelvic CT images provide data in a grayscale image that was formed at the time of scanning. DOSXYZnrc cannot read this data, so it must be converted into the required information by DOSXYZnrc using -. The virtual phantom display with DICOMan Viewer and the virtual phantom display generated by ct-create are shown in Fig. 3.

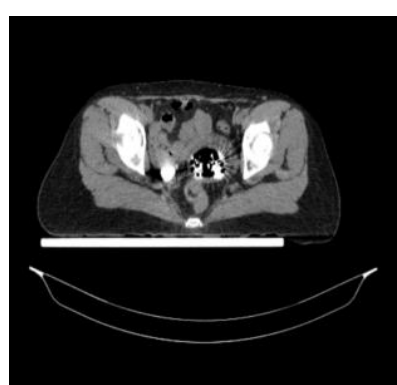

(a)

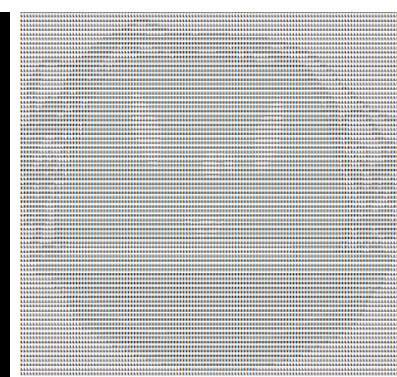

(b)
333333333333333333333322221111112211111
333333333333333333333332222211111222111
33333333333333333333333222221111112211
33333333333333333333333321222211111122
333333333334443333333333331222211111112
33333333334444333333333333122221111111
333333333444443333333333333222221111222
33333334444444333333333333322221122222
33333344444443333333333333322221122111
333334444444443333333333333332221221111
33333444444444333333333333332222221111
333334444444443333333333333332222221111
33333444444444333333333333333222122211
33333444444443333333333333333222222222
333334444444433333333333333333322212112
33333444444443333333333333333322221211

(c)

Fig. 3. (a). Axial phantom view visualized using DICOMan Viewer; (b). The axial phantom fragment, which had changed using ct-create in numeric form; (c). The enlargement of the axial phantom slice.

Based on Fig. 3, the numbers contained in the virtual phantom of the CT image in a numerical form consist of a scale 1 to 4 . It shows the distribution of $\mathrm{HU}$ ranges to distinguish four types of material and the density of different phantom compilers. Number 1 with the HU range from -1000 to -950 represents the air material, number 2 with the $\mathrm{HU}$ range from -950 to -700 represents the lung material, number 3 with the HU range from -700 to +100 represents the soft tissue material, and number 4 with the HU range from +100 to +1600 represents the bone material.

\section{The dose distribution from Ir-192 source for model A}

The dose distribution from the Ir-192 source was analyzed using an isodose curve in the XY plane or axial view. This isodose curve is a combination of 14 Ir-192 sources combined using the Matlab program. The isodose curve of the simulation results was then compared with the TPS results' isodose curve, as shown in Fig. 4. 


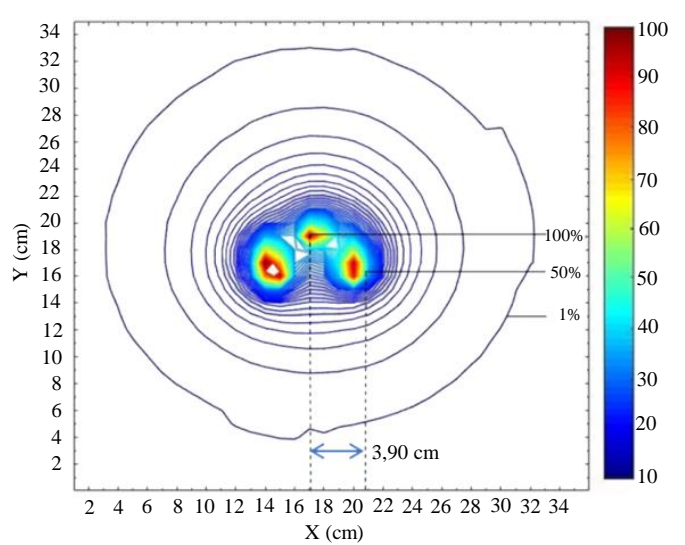

(a)

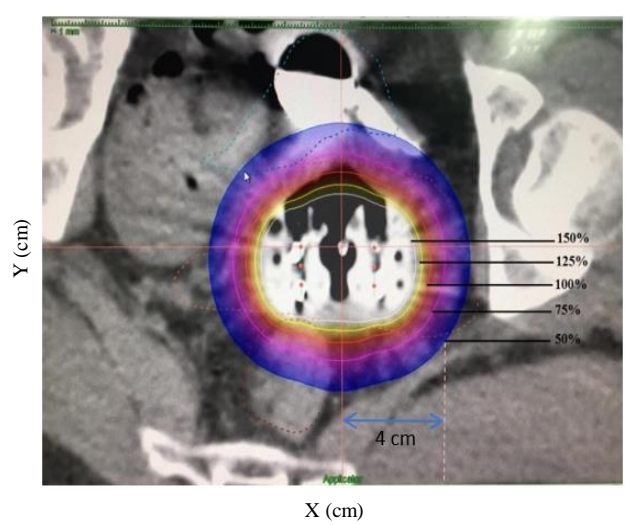

(b)

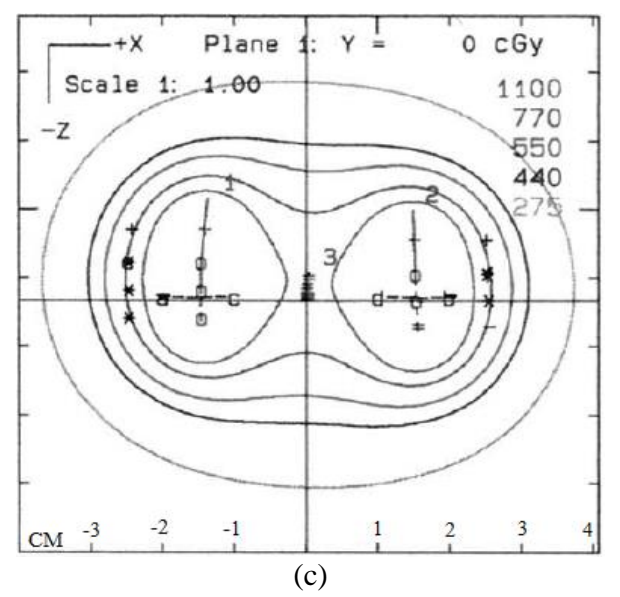

Fig. 4. The isodose curve of the Ir-192 source in the XY plane base on TPS data; (a). With the matlab program; (b). TPS results; (c). Reference results [9].

Based on Fig. 4, the isodose curve in the Manchester system for brachytherapy in the case of cervical cancer from the simulation results, TPS results, and reference results are almost similar quantitatively to each other. The dose distribution pattern in the XY plane or axial view is an ellipse that adapts to the shape of the organ affected by cancer, in this case, the cervix. This pattern provides the optimal dose distribution for destroying cancer cells.
The maximum dose distribution based on the TPS results' isodose curve is shown at a dose of $150 \%$. The minimum dose distribution is shown at the dose of $50 \%$, with the dose distribution range reaching a point of $4 \mathrm{~cm}$ from the center of the isodose curve. The minimum dose distribution based on the reference result shows that the isodose curve has a dose distribution range of approximately $4 \mathrm{~cm}$ from the center of the curve [9]. In contrast to the TPS results and reference results, the maximum dose distribution of the simulated isodose curve is shown at the dose of $100 \%$. The minimum dose distribution is shown at the dose of $1 \%$, with the dose distribution range reaching a point of $15 \mathrm{~cm}$ from the center of the curve. In this case, the center of the curve represents the location of the Ir-192 source contained in the tandem. The $50 \%$ dose distribution range on the isodose curve of the simulation result reached a point of $3.9 \mathrm{~cm}$ from the center of the isodose curve. This result is quantitatively close to the TPS result, with a deviation of $2.5 \%$. Referring to the AAPM REPORT, the dose distribution with a deviation of less than $5 \%$ is still within the tolerance limit.

\section{Comparison of the dose distribution from Ir-192 source for model A and model B}

In the second simulation stage, dose distribution of Ir-192 source were to be optimized with the distance between the sources of $0.5 \mathrm{~cm}$. The simulation results were analyzed using the isodose curve and gamma index in the XY plane or axial view to observe the Ir-192 source dose distribution. The difference between the isodose curve in model A and model B as well as the gamma index is shown in Fig. 5.

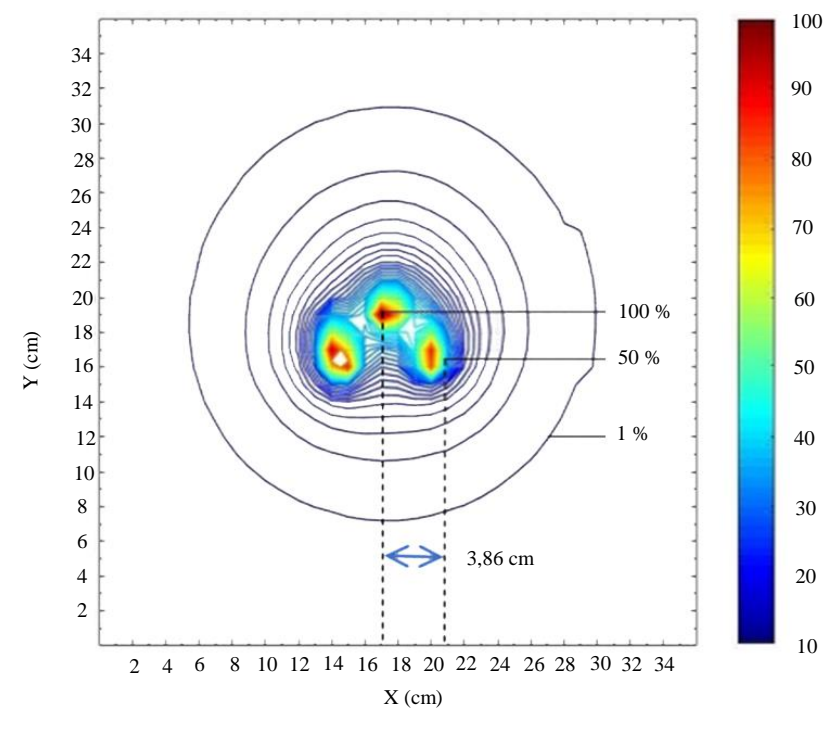

(a) 


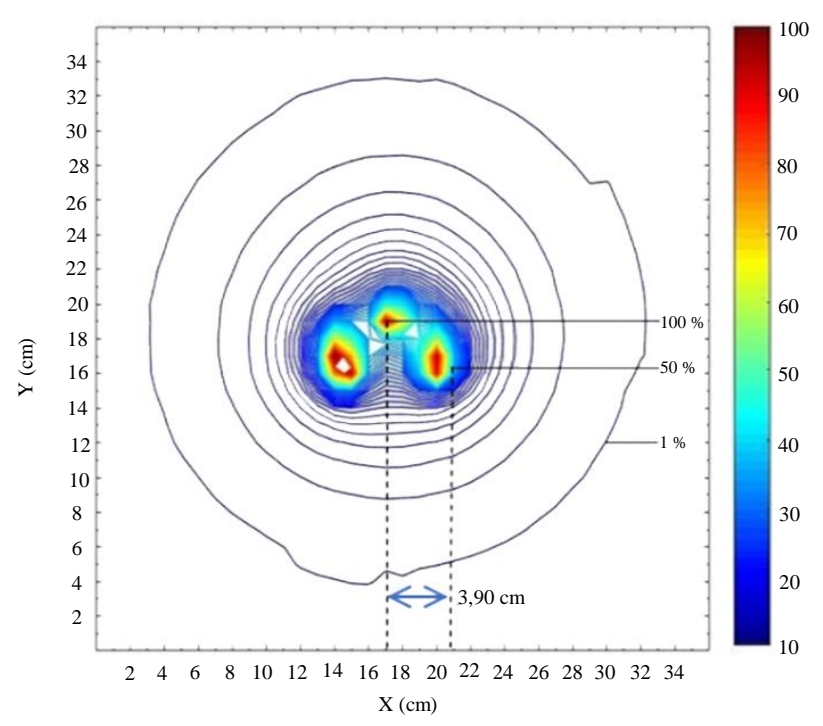

(b)

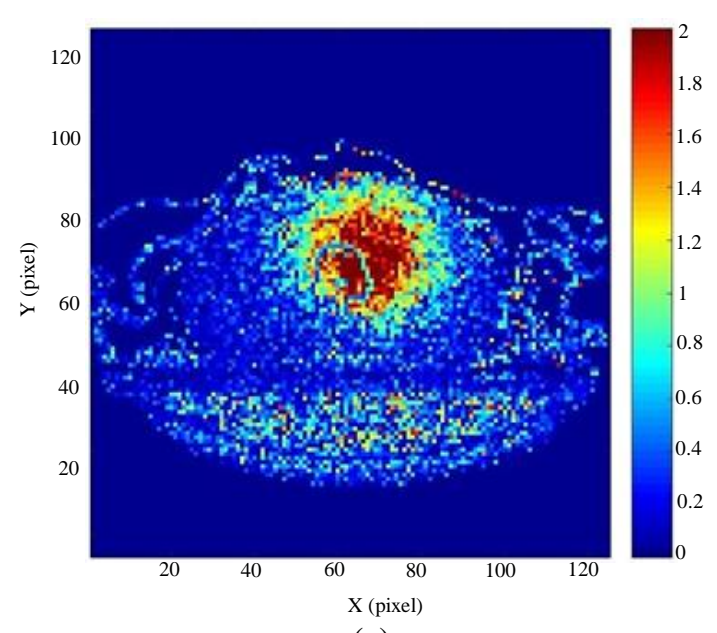

(c)

Fig. 5. The results of the dose distribution from the Ir-192 source in the XY plane; (a). isodose curve in model B;

(b). Isodose curve in model A; (c). The gamma index value of model A and model B.

The isodose curve in Fig. 5 shows the normalized value of dose distribution against the maximum dose value. The curve shows that the full dose distribution is at $100 \%$ dose, while the minimum dose distribution is at $1 \%$. The $50 \%$ dose distribution range for model $\mathrm{A}$ and model $\mathrm{B}$ has a difference the case that, in model $\mathrm{A}$, it reached a point of $3.9 \mathrm{~cm}$. Meanwhile, in model B, it reached the point of $3.86 \mathrm{~cm}$, resulting in a deviation of $1.02 \%$, which is still within the tolerance limit. The isodose curves for model A and model B find on the 40th slice with the isocenter point on the target.

To look deeper into the differences in dose distribution, a $\gamma$-index analysis was performed. Fig. 5.c shows that the resulting $\gamma$-index value for the $50 \%$ dose distribution is 2.26 . The $\gamma$-index value $\geq 1$ indicates a difference in distribution. Also, the percentage of similarity and difference in dose distribution were observed based on the GPR value.
The GPR value is the value that states the ratio of the number of pixels with $\gamma$-index $\leq 1$. The GPR value of the analysis results between model $\mathrm{A}$ and model $\mathrm{B}$ in the entire area is $94.13 \%$, meaning that the GPR value is below $95 \%$, which indicates a difference in dose distribution. Therefore, in this case, the source's location, namely the distance between radioactive sources, is an essential factor in treatment planning in brachytherapy based on the Manchester system. The size of the distance between sources will affect the range of the dose distribution. The distance between sources optimized in model B provides a shorter dose distribution range with a relatively higher uniform dose distribution at the isocenter point on the target.

\section{CONCLUSION}

The isodose curve of the Manchester system for brachytherapy in cases of cervical cancer from the simulation results, TPS results, and reference results show the range of different dose distributions. The dose distribution difference between the Monte Carlo simulation results (Model A) and the TPS results was shown in the dose distribution of $50 \%$. The dose distribution range of the simulation results of Model A reached $3.9 \mathrm{~cm}$ from the center of the isodose curve. Meanwhile, the dose distribution of the TPS result reached a point $4 \mathrm{~cm}$ from the isodose curve center. It results in a deviation value of $2.5 \%$, which is still within the tolerance limit. In model A and model B, there are differences in dose distribution. The difference is from the two models' isodose curves having a different dose distribution range of $50 \%$, namely, in model A, it reached a point of $3.9 \mathrm{~cm}$, while in model $\mathrm{B}$, it reached the point of $3.86 \mathrm{~cm}$, resulting in a deviation of $1.02 \%$, which is still within the tolerance limit. The $\gamma$-index value generated at the $50 \%$ dose distribution was 2.26 . The $\gamma$-index value $\geq 1$ indicates a difference in distribution, while the GPR value in the whole area is $94.13 \%$. It means that the GPR value is below $95 \%$, which indicates a difference in dose distribution.

\section{ACKNOWLEDGMENT}

This study was fully supported by Program Penelitian Kolaborasi Indonesia (PPKI) (grant number 020/WCU-ITB/LL/I/2020).

\section{AUTHOR CONTRIBUTION}

Fitri Kurniati, Fitria Penta Krisna, Junios, and Freddy Haryanto equally contributed as the main contributors to this paper. All authors read and approved the final version of the paper. 


\section{REFERENCES}

1. F. Bray, J. Ferlay, I. Soerjomataram et al., CA Cancer J. Clin. 68 (2018) 394.

2. E. B. Podgorsak, Radiation Oncology Physics: A Handbook for Teachers and Students, International Atomic Energy Agency, Vienna (2005) 210.

3. Junios, Sainstek: Jurnal Sains dan Teknologi 4 (2012) 26. (in Indonesian)

4. Junios and D. Kariman, Jurnal Iptek Terapan $\mathbf{1 0}$ (2016) 155. (in Indonesian)

5. F. P. Krisna, S. Yani, Junios et al., J. Phys. Conf. Ser. 1505 (2020) 012051.

6. S. S. O. Fonseca-Rodrigues, M. Begalli, P. P. Q. Filho et al., Monte Carlo simulation of an Ir-192 brachytherapy source spectra, geometry and anysotropy factors using Geant4 Code, Proceedings of the 2009 IEEE Nuclear Science Symposium Conference Record (NSS/MIC) (2009) 544.

7. A. Sharma, Manchester System For Gynecological Applications (2018).

8. N. Papanikolaou, J. J. Battista, A. L. Boyer et al., Tissue Inhomogeneity Corrections for Megavoltage Photon Beams, in: AAPM Report No. 85, Medical Physics Publishing, U.S.A. (2004) 1.
9. M. Saito, Radiol. Phys. Technol. 12 (2019) 105.

10. A. Manikandan, B. Sarkar, V. T. Rajendran et al., J. Med. Phys. 38 (2013) 148.

11. N. P. Patel, B. Majumdar, P. K. Hota et al., J. Cancer Res. Ther. 1 (2005) 84.

12. Junios, I. Irhas, N. Novitrian et al., Radiol. Phys. Technol. 13 (2020) 398.

13. B. Walters, I. Kawrakow, and D.W.O. Rogers, DOSXYZnrc Users Manual, in: NRCC Report PIRS-794revB, Ionizing Radiation Standards National Research Council of Canada, Ottawa (2011) 130.

14. J. E. Gentle, Statistic and Computing, in: Random Number Generation and Monte Carlo Methods, 2nd ed., Springer Science Business Media Inc, U.S.A. (2003) 25.

15. I. Kawrakow, E. Mainegra-Hing, D. W. O. Rogers et al., The EGSnrc Code System: Monte Carlo Simulation of Electron and Photon Transport, in: NRCC Report PIRS-701, NRC Canada, Ottawa (2011) 125.

16. H. Moseley, NCRP report no. 119. A practical guide to the determination of human exposure to radiofrequency fields: National Council on Radiation Protection and Measurements, Bethesda, U.S.A. (1993) 397. 